\title{
Management of Urinary Tract Infections in Children
}

\author{
Natalie Schellack, ${ }^{1}$ Cahlia Naested, ${ }^{2}$ Nicolene van der Sandt, ${ }^{3}$ Neelaveni Padayachee ${ }^{4}$ \\ ${ }^{1}$ Associate Professor, School of Pharmacy, Faculty of Health Care Sciences, Sefako Makgatho Health Sciences University \\ ${ }^{2}$ Pharmacist at Netcare Greenacres Hospital \\ ${ }^{3}$ Community Service Pharmacist, Madibeng Sub-District Pharmacy, Department of Health, North-West Province \\ 4 Pharmacy and Pharmacology, University of Witwatersrand \\ *Corresponding author, email: natalie.schellack@smu.ac.za
}

\begin{abstract}
Urinary Tract Infections (UTIs) are a common occurrence in paediatrics. UTIs present in children as fever, anorexia, vomiting, lethargy and dysuria. Approximately $80 \%$ of the time, Escherichia coli is the causative bacteria in paediatrics, however, fungal UTI caused by Candida species can occur in premature infants. With an estimated 150 million UTls occurring worldwide annually, this paper aims to establish the ideal management of urinary tract infections in paediatrics. Clinical signs and symptoms of UTI in paediatrics are dependent on age of the child. Neonates (0-27 days old) present with sepsis, vomiting, fever, and prolonged jaundice, while school aged children present with symptoms similar to adults such as dysuria and urgency. Diagnosis of a UTI can be done by using a urine dipstick or using the midstream clean catch method in toilet trained children, and using the transurethral catheterisation or suprapubic aspiration method for infants and young children. In the wake of antibiotic resistance, choosing the best anti-microbial agent for treatment is imperative. Whilst asymptomatic bacteriuria does not require antibiotic treatment, amoxicillin and clavulanic acid combination, cephalexin, cefixime and cefpodoxime are the preferred oral antibiotics, provided there are no known allergies. Ceftriaxone, ampicillin, cefotaxime and gentamycin are the recommended parenteral antibiotics, provided age, allergic status and renal function are considered prior to use. Careful consideration needs to be given before using prophylaxis in UTIs and should be reserved for extreme cases.
\end{abstract}

Keywords: urinary tract infection (UTI), paediatrics, antibiotics, sepsis, cystitis, pyelonephritis

\section{Introduction}

Urinary tract infection (UTI) is defined as a significant growth of bacteria in the urine, together with fever, lethargy, dysuria, pain, anorexia, vomiting and possible kidney scarring. ${ }^{1,2,3}$ UTIs are usually caused by gram-negative aerobic bacilli; approximately $80 \%$ are caused by Escherichia coli.2, Other causative enterobacteria include Enterobacter, Citrobacter, Proteas, Providencia, Morganella and Serratia. ${ }^{4}$ Proteus is common in boys and in children with renal stones. ${ }^{5}$ Coagulase-negative Staphylococci have also been isolated. ${ }^{5}$ Premature infants can also develop a fungal UTI, usually caused by Candida species. ${ }^{4}$

\section{Classification of UTIs}

Urinary tract infections may be classified as follows:

- Asymptomatic bacteriuria: is the absence of clinical signs and symptoms with the presence of bacteriuria.

- Cystitis: localised to the urethra and bladder.Dysuria, frequency, urgency, cloudy urine and lower abdominal discomfort are diagnostic symptoms, while pyuria and haematuria can also occur. This condition is common in girls over two years of age.

- Acute pyelonephritis (AP): presents systemically as high fever, malaise, vomiting, abdominal and loin pain and tenderness, poor feeding and irritability in infants. ${ }^{6} \mathrm{AP}$ is a serious bacterial illness and has a high risk of sepsis and meningitis in young children. ${ }^{7}$

- Uncomplicated UTI: infection is limited to the lower urinary tract and there are no associated urological anomalies. Symptoms include dysuria, frequency, urgency, cloudy urine and abdominal discomfort. Urine dipsticks tests are positive for leukocyte esterase, nitrites and haematuria. ${ }^{8,9}$

- Complicated UTI: an infection of the urinary tract involving the renal parenchyma (AP) or which is associated with underlying congenital anomalies of the kidneys or the urinary tract. It may result in significant short-term morbidity, including septic shock and acute renal failure, especially in infants..$^{8,9}$

UTIs can be a significant source of morbidity in paediatrics and is a common cause of acute illness. ${ }^{1,10}$ Congenital genitourinary conditions, immature host defences, female gender, lack of circumcision in boys, and a prior history of UTI, are all risk factors for UTIs in paediatrics. ${ }^{11}$ According to Alper, $7 \%$ of girls and $2 \%$ of boys will have a UTI that is culture-confirmed and symptomatic by the age of six. ${ }^{5}$

In both sexes, in the first year of life, febrile UTIs show the highest incidence whilst non-febrile UTIs occur predominately 
in girls older than three years of age. ${ }^{12}$ Diagnosing UTI in paediatrics can be a challenge because the clinical presentation may be nonspecific in infants. Invasive methods like urethral catheterization are needed to obtain a reliable urine culture, and for this reason there may be a delay in diagnosis and treatment. ${ }^{13}$ Boys under the age of one have a higher incidence of UTI; however, UTI prevalence increases in girls after the age of one. Renal scarring is thought to develop after a single UTI in approximately $15-41 \%$ of children and chronic kidney disease, proteinuria, hypertension, and complications during pregnancy (if applicable) are a few of the long-term problems. ${ }^{1}$

More than 6 billion dollars in direct healthcare costs are incurred by an estimated 150 million cases of UTI that occur annually worldwide. ${ }^{14}$ With pyelonephritis, younger age, male gender, higher comorbidity status, and concurrent hydronephrosis, stones or sepsis being the strongest predictors for inpatient admission, early signs and symptoms of UTIs in paediatrics need to be identified and treated aggressively to potentially reduce direct healthcare costs. ${ }^{11}$

The ages of paediatric patients were categorised according to the paediatric terminology defined by the National Institute of Child Health and Human Development (NICHD) (2015), developed by Eunice Kennedy Shriver in the USA (Table 1).

Table 1: The 2015 classification of paediatric patients according to the National Institute of Child Health and Human Development

\begin{tabular}{ll}
\hline Classification & Months/Years \\
\hline Neonates & $0-27$ days \\
\hline Infants & Birth to12 months \\
\hline Toddlers & 13 months to 2 years \\
\hline Early childhood & $2-5$ years \\
\hline Middle childhood & $6-11$ years \\
\hline Early adolescence & $12-18$ years \\
\hline Late adolescence & $19-21$ years \\
\hline
\end{tabular}

\section{Pathogenesis}

UTIs typically develop when uropathogens that have colonized the periurethral area ascend via the urethra to the bladder. The pathogens can then spread from the bladder to the kidneys (pyelonephritis) or to the blood stream (bacteraemia). Pathogens can also infect the urinary tract through the direct spread via the faecal-perineal-urethral route..$^{15}$

\section{Clinical signs}

Clinical signs and symptoms depend on the age of the child.

\section{Diagnosis}

A urine dipstick test can be done for screening purposes, and if the dipstick test reveals the presence of leucocytes or nitrites, a clean urine sample should be obtained and sent for microscopy, culture and sensitivity testing. . $^{8,9,16}$

For infants and young children the urine sample should be obtained by transurethral catheterisation or suprapubic aspiration. For older, toilet-trained children, the urine sample can be obtained using the mid-stream clean catch method. ${ }^{4,8}$

Criteria for the diagnosis of a UTI:8,9

Any culture from the suprapubic urine sample

- A culture of $>100$ colonies $/ \mathrm{ml}$ urine of a single organism from a catheter specimen

- A pure culture of $>100$ colonies $/ \mathrm{ml}$ in a midstream clean catch sample

- A consistent culture of a pure growth even with low counts.

A renal ultrasound is recommended for all children who have their first culture-confirmed UTI to assess for structural and functional abnormalities of the urinary tract. Abnormalities include vesicoureteric reflux, posterior urethral valves, ectopic ureters, polycystic kidneys and renal dysplasia. Should the ultrasound reveal an abnormality, a micturating cystourethrogram (MCUG) should be done. ${ }^{4,8,9}$

\section{Treatment}

Treatment should be tailored to the clinical severity, paediatric age, urosepsis suspicion, non-compliance (refusal of fluids, food and/or oral medication) and hydration status (in the case of severe vomiting or diarrhoea). Those with a high risk of serious illness should immediately be referred to a paediatric specialist for optimal care. Also, refer to Figure 1. 15,17-19

Antibiotics are used in the acute management of UTIs, as well as for the prevention of recurrent UTIs. It is important to note that nephrotoxic agents should be avoided where possible. Analgesics may be used to ease voiding symptoms. ${ }^{15,17-19}$

Recommended oral antibiotics typically includes sulfamethoxazole and trimethoprim, amoxicillin/clavulanate, first and second generation cephalosporins and nitrofurantoin (Table 3). Parental agents used in the treatment of paediatric UTIs include ceftriaxone, cefotaxime, ampicillin, gentamicin and tobramycin (Table 4). A list of principles to ensure appropriate

Table 2: Clinical signs and symptoms of UTI

\begin{tabular}{llll}
\hline & Neonates & Infants and young children & School-aged children \\
\hline Signs and symptoms & Prolonged jaundice & Persistent fever & Symptoms similar to adults that present \\
& Sepsis & Dysuria & with UTI, including: \\
& Failure to thrive & Failure to thrive & Dysuria \\
& Vomiting & Strong smelling urine & Urgency \\
& Fever & Haematuria & \\
Renal failure & Abdominal or flank pain & New-onset urinary & \\
& & incontinence & \\
\end{tabular}




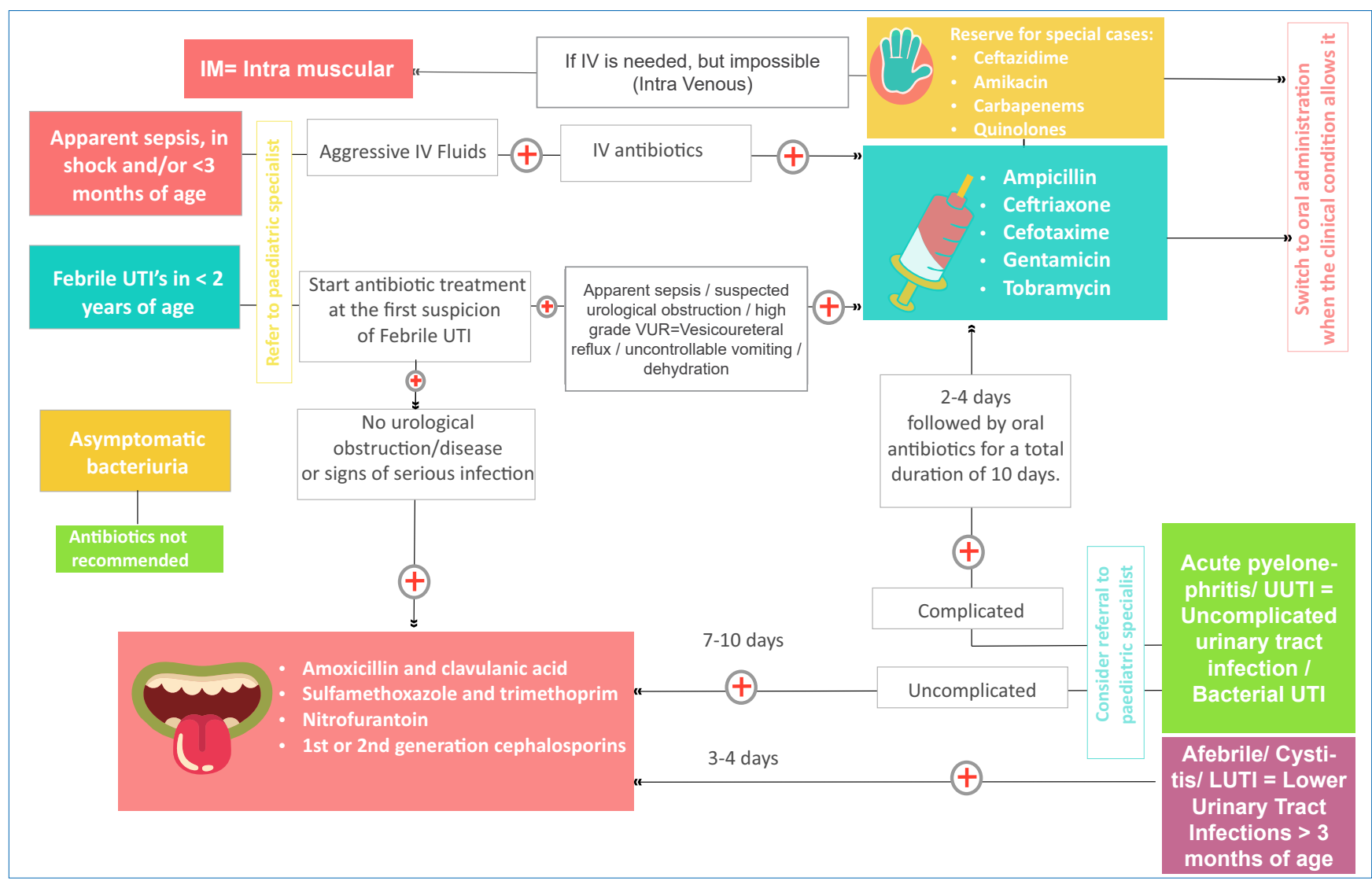

Figure 1: Suggested approach to the management of paediatric UTIs 15,17-19

antibiotic use and an algorithm providing guidance for paediatric UTI treatment per UTI classification can be seen in Table 3.

Table 3: Principles of appropriate for antibiotic use in paediatric UTI care Principles for appropriate antibiotic use in paediatric UTI care Only initiate an antibiotic once a urinalysis has been performed and a urine specimen for culture was obtained.

With the alarming increase in AMR, due care should be taken when choosing an agent.

Local antimicrobial susceptibility patterns should be used when deciding on empiric treatment and should cover the most common uropathogens (Escherichia coli, Enterococcus, Proteus, and Klebsiella species).

Reassess antibiotic 48 hours after initiation and modify according to susceptibility results.

Complicated or uncomplicated UTIs should be treated for 7-10 days. However, short-courses of therapy may be used in adolescent girls with non-toxic UTIs.

If during the use of prophylactic medication a paediatric develops an infection: do not increase dose of current antibiotic, switch to a different agent.

Antibiotic treatment in the case of asymptomatic bacteriuria is not recommended.

$16,17,18$

\section{Analgesics}

Paracetamol can be used to provide relief from voiding symptoms.9,15,20 The recommended dose of paracetamol is $15 \mathrm{mg} / \mathrm{kg} 6$ hourly. Both the World Health Organization and the National Department of Health recommend against the use of
Non-Steroidal Anti-inflammatories (NSAIDs) as an analgesic for children with UTIs.9,20

\section{Antibiotic prophylaxis}

In paediatrics with a single prior UTI, the use of antibiotic prophylaxis is not routinely recommended. In asymptomatic bacteriuria, antibiotic prophylaxis should not be used. Paediatric patients with recurrent UTIs should be thoroughly assessed to rule out any urinary tract abnormalities, where prophylactic antibiotics may be used, based on an individual evaluation. When prescribing prophylactic antibiotics, it is important to consider the ever increasing AMR (antimicrobial resistance) patterns. Local microorganism susceptibility should be taken into consideration when choosing an agent, and preferably a narrow-spectrum antibiotic should be used. Table 6 shows agebased prophylactic antibiotic recommendations .15,17-19 Tables 4 and 5 provides an overview of antibiotics used in the treatment of paediatric UTI

\section{Non-pharmacological interventions ${ }^{18,20}$}

The following interventions may be applied:

- Addressing dysfunctional elimination syndromes and constipation

- Improving bowel habits

- Encouraging adequate hydration

- Not delaying voiding

- Improving general hygiene. 
Table 4: Oral antibiotics used in paediatric UTI care

\begin{tabular}{|c|c|c|}
\hline Oral antibiotics & Dosing & Notes \\
\hline Amoxicillin and clavulanic acid & $\begin{array}{l}20-40 \mathrm{mg} / \mathrm{kg} \text { divided q8h } \\
\text { Uncomplicated UTI: } 30 \mathrm{mg} / \mathrm{kg} / \text { dose } 8 \mathrm{~h} \text { po } \\
\text { Complicated UTI: } 25 \mathrm{mg} / \mathrm{kg} / \text { dose } 8 \mathrm{H} \mathrm{IV} \mathrm{=intra} \mathrm{venous}{ }^{8,9}\end{array}$ & Alternative if allergic/intolerant to macrolides \\
\hline Amoxicillin & Not recommended due to high E.coli resistance & \\
\hline Sulfamethoxazole and trimethoprim & 30-60 mg/kg SMZ, 6-12 mg/kg TMP divided q12 & $\begin{array}{l}\text { Not recommended as an option by South } \\
\text { African guidelines }{ }^{4,8,9}\end{array}$ \\
\hline Cephalexin & $50-100 \mathrm{mg} / \mathrm{kg} /$ day divided $\mathrm{q} 6 \mathrm{~h}$ & \\
\hline Cefixime & $8 \mathrm{mg} / \mathrm{kg} /$ day & \\
\hline Cefpodoxime & $10 \mathrm{mg} / \mathrm{kg} /$ day divided $\mathrm{q} 12 \mathrm{~h}$ & Use in mixed aerobic-anaerobic UTIs \\
\hline Ciprofloxacin & $\begin{array}{l}\text { Not recommended as a first choice in paediatrics } \\
\text { Can be used to treat MDR infections when there is no ot } \\
\text { UTIs caused by Pseudomonas aeruginosa or other MDR g }\end{array}$ & $\begin{array}{l}\text { her safe and effective alternative. Limit to treat } \\
\text { ram-negative bacteria. }{ }^{13}\end{array}$ \\
\hline
\end{tabular}

$\mathrm{SMZ}=$ sulfamethoxazole, $\mathrm{TMP}=$ trimethoprim, $\mathrm{q}=$ every $15,17-19$

Table 5: Parenteral antibiotics used in paediatric UTI care

\begin{tabular}{|c|c|c|}
\hline IV antibiotic & Dosing & Notes \\
\hline Ceftriaxone & $50-75 \mathrm{mg} / \mathrm{kg} /$ day IV/IM single dose or divided q12h & $\begin{array}{l}\text { Contra-indicated in }<6 \text { weeks of age; bilirubin may be } \\
\text { displaced from albumin }\end{array}$ \\
\hline Ampicillin & $100 \mathrm{mg} / \mathrm{kg} /$ day IV/IM divided q8h & $\begin{array}{l}<2 \text { weeks of age used in combo with gentamicin } \\
\text { Used in paediatrics allergic to cephalosporins; } \\
\text { Initial therapy where either gram-positive cocci or no } \\
\text { organism is found in urinary sediment }\end{array}$ \\
\hline Cefotaxime & $150 \mathrm{mg} / \mathrm{kg} /$ day IV/IM divided q6-8h & $\begin{array}{l}\text { Safe in }<6 \text { weeks of age } \\
\text { Aged } 2-8 \text { weeks: Used in combo with ampicillin; } \\
\text { Use in neonates and jaundiced paediatrics }\end{array}$ \\
\hline Gentamicin & $\begin{array}{l}<7 \text { days: } 3.5-5 \mathrm{mg} / \mathrm{kg} / \text { dose IV q24h } \\
<5 \text { years: } 2.5 \mathrm{mg} / \mathrm{kg} / \text { dose IV q8h or single daily dosing with } \\
\text { normal renal function of } 5-7.5 \mathrm{mg} / \mathrm{kg} / \text { dose IV q24h } \\
\geq 5 \text { y: } 2-2.5 \mathrm{mg} / \mathrm{kg} / \mathrm{dose} I V \mathrm{q} 8 \mathrm{~h} \text { or single daily dosing with } \\
\text { normal renal function; } 5-7.5 \mathrm{mg} / \mathrm{kg} / \text { dose IV q } 24 \mathrm{~h}\end{array}$ & $\begin{array}{l}\text { Monitor blood levels and kidney function if therapy extends } \\
>48 \text { hours; } \\
\text { Empirical therapy should not be used for }>3 \text { days. If } \\
\text { empirical therapy is still required, consider changing to } \\
\text { ceftriaxone }\end{array}$ \\
\hline
\end{tabular}

IV = Intravascular, IM= Intramuscular, q= every $15,17-19$

Table 6: Prophylactic antibiotic recommendations

\begin{tabular}{|c|c|c|}
\hline Age & Antibiotic & Dosage \\
\hline$<2$ months of age & \multirow{3}{*}{$\begin{array}{l}\text { Amoxicillin }{ }^{* *} \\
\text { 1st/2nd generation Cephalosporin } \\
* * *\end{array}$} & \multirow{3}{*}{ A $1 / 3$ or $1 / 4$ of normal recommended dose } \\
\hline$<8$ weeks of age & & \\
\hline If nitrofurantoin/ $\mathrm{TI}$ & & \\
\hline$>2$ months & SMZ-TMP & $5-10 \mathrm{mg} / \mathrm{kg} /$ day SMZ, 1-2 mg/kg/day TMP \\
\hline$>2-3$ years of age & Nitrofurantoin & $1-2$ mg/kg/day. \\
\hline
\end{tabular}

\section{Preventative measures}

The use of vitamin C, cranberry juice, vaccines with uropathogenic strains and/or probiotics is not recommended due to a lack of scientific evidence. ${ }^{21}$

\section{Referral}

Referral should be considered in the following circumstances ${ }^{8,9}$ :

- Poor response, despite adequate treatment (persistent positive culture or fever)

- Recurrent UTIs,

- Suspected obstruction or abnormal imaging

- Abnormal renal function

\section{Conclusion}

UTI is an uncomfortable and unpleasant condition, which requires immediate attention in paediatrics. Adequate urine tests are necessary to isolate the bacteria, thus narrowing the choice of antibiotic. Appropriate antibiotic and supportive treatment must be considered to ensure a quick recovery and to prevent complications like renal scarring in young children. With antibiotic resistance taking centre stage globally, using the most appropriate antibiotic is essential to obtain the best results without compromising the patients' wellbeing. Prior to choosing an antibiotic, the allergic status, age and renal function must be established. Supportive treatment like paracetamol together 
with non-pharmacological advice, such as good hygiene and adequate hydration, can reduce the discomfort for the patient.

\section{References}

1. Ramlakhan S, Singh V, Stone J, Ramtahal A. Clinical Options for the Treatment of Urinary Tract Infections in Children. Clinical Medicine Insights. Pediatrics. 2014; 8:31-7. Available from: http://doi.org/10.4137/CMPed.S8100

2. Noorbakhsh S, Zarabi V. UTI in Children, Urinary Tract Infections, Tenke P, ed. ISBN: 978-953-307-757-4, InTech; 2011. Available from: http://www.intechopen. com/books/urinary-tractinfections/uti-in-children

3. Keren R, Shaikh N, Pohl H, Gravens-Mueller L, Ivanova A, Zaoutis L, et al. Risk factors for recurrent urinary tract infection and renal scarring. Pediatrics. 2015; peds-2015.

4. Ampath Infectious Diseases Peer Group. Treatment of urinary tract infections, in Guidelines to antimicrobial therapy and the laboratory diagnosis of infectious diseases. 5th ed. AmpathTrust; 2017;438.

5. Alper BS, Curry SH. Urinary Tract Infection in Children. Am Fam Physician. 2005; 15-72(12):2483-8.

6. Strohmeier Y, Hodson EM, Willis NS, Webster AC, Craig JC. Antibiotics for acute pyelonephritis in children. The Cochrane Library. 2014.

7. Morello W, La Scola C, Alberici I, Montini G. Acute pyelonephritis in children. Pediatr Nephrol. 2016;31(8):1253-65.

8. Murray J. Urinary Tract Infections. Paarl Hospital Paediatric Guideline. EMGuidance application. 2015 [accessed 4 Nov 2017].

9. National Department of Health. Standard treatment guidelines and essential medicines list for South Africa, Hospital level paediatrics, 2013. National Department of Health, Pretoria, South Africa; 2013.

10. Robinson JL, Finlay JC, Lang ME, Bortolussi R. Urinary Tract Infections in Infants and Children: Diagnosis and Management. Paediatr Child Health. 2014;19(6):315-9.

11. Sood A, Penna F, Eleswarapu S, Pucheril D, Weaver J, Wagner JC, et al. Incidence, admission rates, and economic burden of pediatric emergency department visits for urinary tract infection: Data from the nationwide emergency department sample, 2006 to 2011. J Pediatr Urol. 2015;11(5):246-e1.

12. Montini G, Tullus K, Hewitt I. Febrile Urinary Tract Infections in Children. N Engl J Med. 2011;365:239-50.

13. Roberts KB. Urinary tract infection: clinical practice guideline for the diagnosis and management of the initial UTI in febrile infants and children 2 to 24 months. American Academy of Paediatrics. 2011;595-610.

14. Hanna-Wakim RH, Ghanem ST, El Helou MW, et al. Epidemiology and characteristics of urinary tract infections in children and adolescents. Front Cell Infect Microbiol. 2015;5(45).

15. Fisher D. Pediatric urinary tract infections. 2017 [accessed on 4 November 2017] Available from: https://emedicine.medscape.com/article/969643-overview\#a4

16. White B. Diagnosis and treatment of urinary tract infections in children. Am Fam Physician. 2011;83(4):409-15.

17. Cdc.gov. Pediatric Treatment Recommendations | Community | Antibiotic Use | CDC [online]. 2017 [accessed on 3 Nov 2017]. Available from: https://www.cdc. gov/antibiotic-use/community/for-hcp/outpatient-hcp/pediatric-treatment-rec. html

18. Nice.org.uk. Urinary tract infection in under 16s: diagnosis and management | Guidance and guidelines | NICE [online]. 2007 [accessed on 3 Nov 2017]. Available from: https://www.nice.org.uk/guidance/cg54/chapter/ Recommendations\#acute-management

19. Tekgül S, Dogan H, Kočvara R, Nijman J, Radmayr C, Stein R. Paediatric Urology Uroweb [online]. 2017 [accessed on 3 Nov 2017]. Available from: http://uroweb. org/guideline/paediatric-urology/?type=pocket-guidelines

20. World Health Organization. Pocket book of hospital care for children: Guidelines for the management of common childhood illnesses, 2nd edition. World Health Organization; 2013.

21. SNS. Clinical Practice Guideline for Urinary Tract Infection in Children. SNS Ministry of Science and Research [ebook]. 2011 [accessed 3 Nov 2017];12360. Available from: http://www.guiasalud.es/GPC/GPC_483_ITU_poblacion pediatrica_ICS_ing.pdf 